\title{
A MICROMECHANICAL DAMAGE THEORY FOR BRITTLE MATERIALS WITH SMALL CRACKS
}

\author{
S. ZHAN, T.-C. WANG, and X. HAN \\ LNM, Institute of Mechanics, Chinese Academy of Sciences, Beijing 100080, People's Republic of China \\ Abstract-For brittle solids containing numerous small cracks, a micromechanical damage theory is \\ presented which accounts for the interactions between different small cracks and the effect of the boundary \\ of a finite solid, and includes growth of the pre-existing small cracks. The analysis is based on a \\ superposition scheme and series expansions of the complex potentials. The small crack evolution process \\ is simulated through the use of fracture mechanics incorporating appropriate failure criteria. The \\ stress-strain relations are obtained from the micromechanics analysis. Typical examples are given to \\ illustrate the potential capability of the proposed theory. These results show that the present method \\ provides a direct and efficient approach to deal with brittle finite solids containing multiple small cracks. \\ The stress-strain relation curves are evaluated for a rectangular plate containing small cracks.
}

Keywords-Micromechanics; Damage; Brittle materials; Small cracks; Evolution process.

\section{NOMENCLATURE}

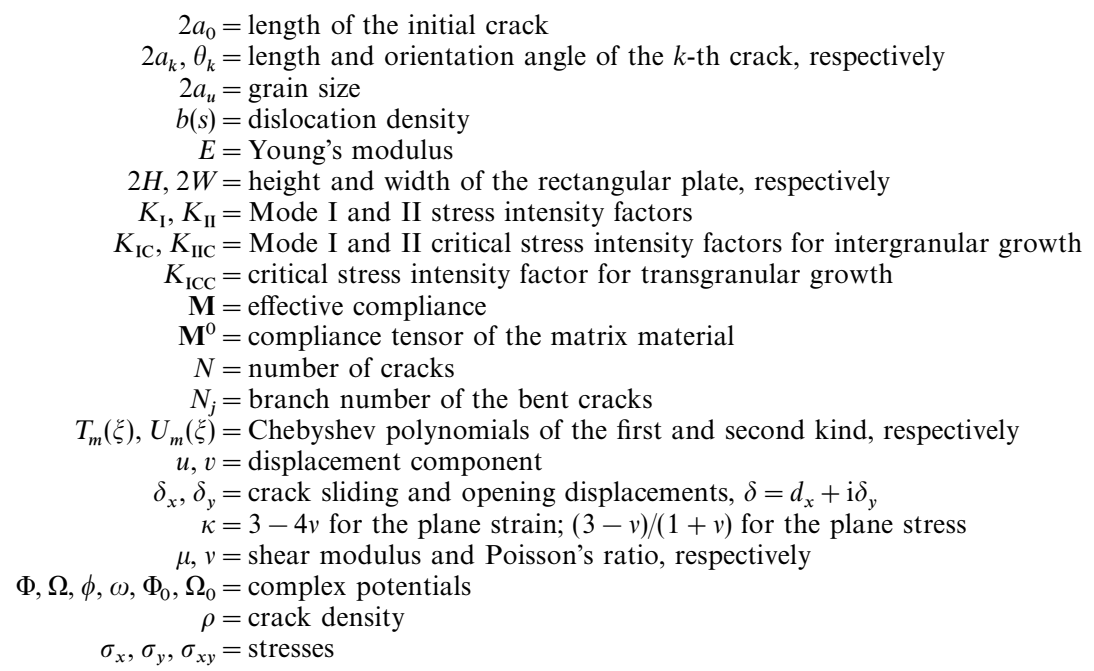

\section{INTRODUCTION}

Ceramics matrix and metal matrix composites, ceramics, rocks, concrete, and other brittle materials often contain large numbers of small cracks. Due to the presence, growth and nucleation of small cracks, the non-linear mechanical responses of damaged solids are of significant importance to engineers, and have been the subject of many investigations. In the current literature, there are several micromechanics stationary damage models to estimate the effective moduli of solids containing small cracks, e.g. the dilute or non-interacting solution, the self-consistent method [1], the generalized self-consistent model [2], the differential scheme [3] and the Mori-Tanaka method [4]. In these models, small crack interactions are entirely neglected or indirectly accounted. These methods are only valid for low or moderate crack density, since they do not depend on locations 
of small cracks. As crack density increases and small crack spacings are closer, strong interactions between small cracks occur, and the mutual positions of cracks become important. Then, strong small crack interaction models have been proposed. Kachanov [5] proposed a pseudo-traction method to solve the multiple cracks problem. This method is simple, and can be used for higher small crack concentrations. Huang et al. [6] used a hybrid BEM method, in conjunction with a unit cell model, to calculate the effective moduli of microcracked solids. A unit cell, which can be considered as a representation block in the solid, is assumed to be periodic in the solid so as to account for interactions between cracks inside and outside the cell. It is noted that the foregoing methods are only used in infinite media, and they do not deal with the damage and fracture process of brittle materials. In general, a tensile stress-strain curve often includes the stages of linear elasticity, pre-peak non-linear hardening, tension softening, etc. Krajcinovic and Fanella [7], Ju and Lee [8], and others presented the micromechanical damage models for microcrackweakened brittle materials. Because their microcrack kinetic algorithms based on fracture criteria are intrinsically stress-controlled, these models are limited to the pre-peak non-linear hardening regime. In addition, the damaged compliance tensor is derived by using the self-consistent method or Taylor's model, so these micromechanical evolutionary damage models are only valid for weak small crack interactions under low or at most moderate small crack concentrations. Ortiz [9], Huang and Karihaloo [10], and Horii et al. [11] also developed several models to describe the intrinsic tension softening behaviour of quasi-brittle materials. Chen and Yamaguchi [12] simulated the softening response by the finite element method considering microstructures of concrete materials. Feng and $\mathrm{Yu}$ [13] gave the complete stress-strain relation by using the concept of domain of microcrack growth (DMG) without considering interaction between microcracks.

The purpose of this work is to present a micromechanical damage theory to study the damage evolution process of brittle or semi-brittle materials. The theory directly accounts for the interactions between different small cracks and the effect of outer boundary of a finite plate, and gives the complete stress-strain relation curve. An outline of this work is as follows. Below, a general method is presented for solving the plane elasticity problem of finite plates with multiple small cracks. Analysis is based on superposition scheme and series expansions of the complex potentials. By using the traction-free conditions on each crack surface and boundary conditions along the outer boundary, a set of governing equations is formulated. The governing equations are solved numerically on the basis of a boundary collocation procedure. When the algebraic equations are solved, the complex potentials and stress components produced by each crack and the outer boundary are known. According to the superposition principle, the stress fields of the finite plate are obtained with the aid of the transformation formulae from the local coordinate systems into the global one. Then, the small crack evolution process is simulated through the use of fracture mechanics incorporating appropriate failure criteria. A complete stress-strain curve is given. Finally, typical examples are given to illustrate the potential capability of the proposed theory. Overall, non-linear stress-strain curves are evaluated for rectangular plates with small cracks.

\section{MICROMECHANICAL DAMAGE THEORY}

\section{Basic formulae}

\section{A single crack}

It is well known that stresses and displacements for a homogeneous elastic body under plane deformation can be represented by two complex potentials. For convenience, potentials $\Phi(z)$ and 
$\Omega(z)$ will be used. Stresses can be derived from [14]

$$
\begin{gathered}
\sigma_{x}+\sigma_{y}=2[\Phi(z)+\overline{\Phi(z)}] \\
\sigma_{y}-\mathrm{i} \sigma_{x y}=\Phi(z)+\Omega(\bar{z})+(z-\bar{z}) \overline{\Phi^{\prime}(z)}
\end{gathered}
$$

A crack can be considered as a continuous distribution of infinitesimal edge dislocation. For a single crack lying on the real axis between $s=-a$ and $s=a$ in an infinite plate, the complex potentials $\Phi(z)$ and $\Omega(z)$ are given by the following formula:

$$
\Phi(z)=\Omega(z)=\frac{\mu}{\pi \mathrm{i}(\kappa+1)} \int_{-a}^{a} \frac{b(s)}{z-s} \mathrm{~d} s
$$

where $b(s)$ is the dislocation density at point $z=s$ on the crack surface. For the plane strain problem $\kappa=3-4 v$, and plane stress problem $\kappa=(3-v) /(1+v) . \mu$ is the shear modulus and $v$ is Poisson's ratio.

The dislocation density can be expressed as the following series [16]:

$$
\frac{\mu}{\mathrm{i}(\kappa+1)} b(s)=-\frac{\mathrm{d} \delta(s)}{\mathrm{d} s}=\sum_{m=0}^{\infty} \alpha_{m} \frac{T_{m}(\xi)}{\sqrt{1-\xi^{2}}}
$$

where $\delta=\delta_{x}+\mathrm{i} \delta_{y}, \delta_{x}$ is the crack sliding displacement, and $\delta_{y}$ is the crack opening displacement (COD). $T_{m}(\xi)$ is a Chebyshev polynomial of the first kind, $\xi=s / a$.

Then, substituting Eq. (3) into Eq. (2), we obtain

$$
\Phi(z)=\Omega(z)=\sum_{m=0}^{\infty} \alpha_{m}\left(\frac{z}{a}-\sqrt{\frac{z^{2}}{a^{2}}-1}\right)^{m} / \sqrt{\frac{z^{2}}{a^{2}}-1}
$$

In the derivative of Eq. (4), the following formula is used,

$$
\frac{1}{\pi} \int_{-1}^{1} \frac{\left(1-\xi^{2}\right)^{-1 / 2} T_{m}(\xi) \mathrm{d} \xi}{z-\xi}=\left(z-\sqrt{z^{2}-1}\right)^{m} / \sqrt{z^{2}-1}, \quad m=0,1,2, \ldots
$$

Eq. (4) has been independently proposed by Gross [15], and Han and Wang [16] from different viewpoints.

Substituting Eq. (4) into Eq. (1), the stress field at any point due to the crack can be expressed as a series. Specially, the stress field on the crack surface can be expressed as

$$
\sigma_{y}-\mathrm{i} \sigma_{x y}=\Phi^{+}(s)+\Omega^{-}(s)=-2 \sum_{m=0}^{\infty} \alpha_{m} U_{m-1}(s / a)
$$

where $U_{m}(s / a)$ is a Chebyshev polynomial of the second kind.

The unknown coefficients $\alpha_{m}$ need to be determined.

\section{$A$ set of arbitrary cracks}

A system containing a set of arbitrary 2D $N$ cracks in an infinite plate is shown in Fig. 1. A global Cartesian coordinate system $O x y$ is situated. A local normal-tangential coordinate system employed with origins $\left(O_{k}\right)$ at the centre of the $k$-th crack is represented by $x_{k}$ and $y_{k}$. The geometry of the $k$-th crack is specified by the centre coordinates $\left(x_{\mathrm{c}}^{(k)}, y_{\mathrm{c}}^{(k)}\right)$, orientation angle $\theta_{k}$, and the halflength of the crack $a_{k}$.

The stresses produced by the $k$-th crack opening and sliding displacements in the local coordinate 


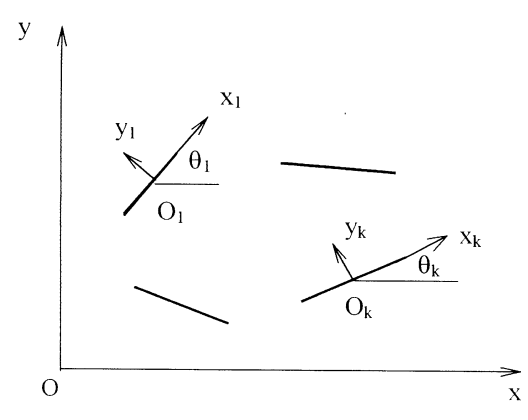

Fig. 1. A set of arbitrary cracks.

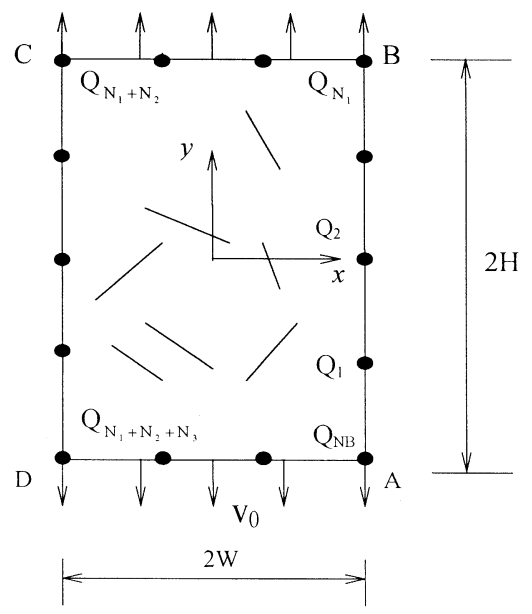

Fig. 2. Tension of rectangular plate with microcracks.

system $O_{k} x_{k} y_{k}$ take the form

$$
\begin{gathered}
\sigma_{x k}+\sigma_{y k}=2\left[\Phi_{k}\left(z_{k}\right)+\overline{\Phi_{k}\left(z_{k}\right)}\right] \\
\sigma_{y k}-\mathrm{i} \sigma_{x y k}=\Phi_{k}\left(z_{k}\right)+\Omega_{k}\left(\overline{z_{k}}\right)+\left(z_{k}-\overline{z_{k}}\right) \overline{\Phi_{k}^{\prime}\left(z_{k}\right)}
\end{gathered}
$$

where

$$
\begin{gathered}
\Phi_{k}\left(z_{k}\right)=\Omega_{k}\left(z_{k}\right)=\sum_{m=0}^{\infty} \alpha_{k m}\left(\frac{z_{k}}{a_{k}}-\sqrt{\frac{z_{k}^{2}}{a_{k}^{2}}-1}\right)^{m} / \sqrt{\frac{z_{k}^{2}}{a_{k}^{2}}-1} \\
z_{k}=x_{k}+\mathrm{i} y_{k}=\left(z-C_{k}\right) \mathrm{e}^{-\mathrm{i} \theta_{k}} \\
z=x+\mathrm{i} y, \quad C_{k}=x_{\mathrm{c}}^{(k)}+\mathrm{i} y_{\mathrm{c}}^{(k)}
\end{gathered}
$$

and on the $k$-th crack surface

$$
\sigma_{y k}^{(k)}\left(z_{k}\right)-\mathrm{i} \sigma_{x y k}^{(k)}\left(x_{k}\right)=\Phi^{+}\left(x_{k}\right)+\Omega^{-}\left(x_{k}\right)=-2 \sum_{m=0}^{\infty} \alpha_{k m} U_{m-1}\left(x_{k} / a_{k}\right)
$$

According to the formulae of coordinate system transformation, the tractions on the $l$-th crack surface in the local coordinate system $O_{l} x_{l} y_{l}$ produced by the $k$-th crack can be written as follows

$$
\sigma_{y l}^{(k)}\left(z_{l}\right)-\mathrm{i} \sigma_{x y l}^{(k)}\left(z_{l}\right)=\frac{1}{2}\left[\sigma_{x k}\left(z_{k}\right)+\sigma_{y k}\left(z_{k}\right)\right]\left(1-\mathrm{e}^{-2 \mathrm{i} \theta}\right)+\left[\sigma_{y k}\left(z_{k}\right)-\mathrm{i} \sigma_{x y k}\left(z_{k}\right)\right] \mathrm{e}^{-2 \mathrm{i} \theta}, \quad k=1,2, \ldots, N
$$

where

$$
\theta=\theta_{l}-\theta_{k}, \quad z_{k}=\left(C_{l}-C_{k}\right) \mathrm{e}^{-\mathrm{i} \theta_{k}}+z_{l} \mathrm{e}^{\mathrm{i}\left(\theta_{l}-\theta_{k}\right)}
$$

Solution for rectangular plate under tension (uniform displacement without shear)

According to the superposition scheme, the problem of finite plate with small cracks can be decomposed into two subproblems. The first subproblem is the interaction problem of numerous small cracks within an assigned finite region in an infinite plate and no stress is applied at infinity. 
The geometry configurations of the small cracks, and the opening and sliding displacements of each small crack inside the assigned finite region are the same as that of the original problem. The basic formulae in the first subproblem have been derived in the last subsection. The second subproblem is a homogeneous problem, in which the homogeneous finite plate of the matrix material is subject to loading on the outer boundary. The loading consists of the same external loading as in the original problem and the extra loads that are applied in order to counteract the tractions induced by the small cracks along the outer boundary of the assigned finite region in the first problem, and this satisfies the outer boundary condition of the finite plate. In the second subproblem, the stresses caused by the loads applied at the outer boundary can be expressed by two complex potentials $\Phi_{0}(z)$ and $\Omega_{0}(z)$ :

$$
\begin{gathered}
\sigma_{x}^{(0)}+\sigma_{y}^{(0)}=2\left[\Phi_{0}(z)+\overline{\Phi_{0}(z)}\right] \\
\sigma_{y}^{(0)}-\mathrm{i} \sigma_{x y}^{(0)}=\Phi_{0}(z)+\Omega_{0}(\bar{z})+(z-\bar{z}) \overline{\Phi_{0}^{\prime}(z)}
\end{gathered}
$$

where

$$
\Phi_{0}(z)=\sum_{n=1}^{\infty} n b_{n} z^{n-1}, \quad \Omega_{0}(z)=\sum_{n=1}^{\infty} n c_{n} z^{n-1}
$$

According to the superposition scheme, the traction-free condition on each crack surface can be written as follows

$$
\sigma_{y l}^{(0)}\left(x_{l}\right)-\mathrm{i} \sigma_{x y l}^{(0)}\left(z_{l}\right)+\sum_{k=1}^{N}\left[\sigma_{y l}^{(k)}\left(x_{l}\right)-\mathrm{i} \sigma_{x y l}^{(k)}\left(x_{l}\right)\right]=0, \quad\left|x_{l}\right|<a_{l}, \quad l=1,2, \ldots, N
$$

where $\sigma_{y l}^{(k)}\left(x_{l}\right)-\mathrm{i} \sigma_{x y l}^{(k)}\left(x_{l}\right)$ are the tractions along the $l$-th small crack surface in the local coordinate system $O_{l} x_{l} y_{l}$ produced by the $k$-th small crack in the first subproblem. $\sigma_{y l}^{(0)}\left(x_{l}\right)-\mathrm{i} \sigma_{x y l}^{(0)}\left(x_{l}\right)$ are the tractions along the $l$-th small crack surface in the local coordinate system $O_{l} x_{l} y_{l}$ produced by the loading applied to the outer boundary of the finite plate in the second subproblem.

Consider a rectangular plate with small cracks that is subjected to the external uniaxial tension (uniform displacement without shear); Fig. 2. Point $\mathrm{A}$ is assumed to be fixed at all time, point $\mathrm{A}^{*}$ is permitted to move. The boundary conditions in the present analysis are written in terms of resultant forces from $\mathrm{A}$ to $\mathrm{A}^{*}$, and the displacement is as follows:

$$
\begin{array}{ll}
\mathrm{A}^{*} \in \mathrm{AB}: & X+\mathrm{i} Y=0 \\
\mathrm{~A}^{*} \in \mathrm{BC}: & X=0, \quad v=v_{0} \\
\mathrm{~A}^{*} \in \mathrm{CD}: & X+\mathrm{i} Y=\mathrm{i} Y_{\mathrm{c}} \\
\mathrm{A}^{*} \in \mathrm{DA}: & X=0, \quad v=-v_{0}
\end{array}
$$

where $Y_{\mathrm{c}}$ is the resultant force in the $y$-direction from A to C. In the global coordinate system, the resultant forces from $\mathrm{A}$ to $\mathrm{A}^{*}$ can be expressed as:

$$
\begin{aligned}
X(z)+\mathrm{i} Y(z) & =X_{0}(z)+\mathrm{i} Y_{0}(z)+\sum_{k=1}^{N}\left[X_{k}\left(z_{k}\right)+\mathrm{i} Y_{k}\left(z_{k}\right)\right] \mathrm{e}^{\mathrm{i} \theta_{k}} \\
& =-\mathrm{i}\left[\phi_{0}(z)+\omega_{0}(\bar{z})+(z-\bar{z}) \overline{\Phi_{0}(z)}\right]_{\mathrm{A}}^{\mathrm{A}^{*}}-\sum_{k=1}^{N} \mathrm{i}\left\{\left[\phi_{k}\left(z_{k}\right)+\omega_{k}\left(\overline{z_{k}}\right)+\left(z_{k}-\overline{z_{k}}\right) \overline{\Phi_{k}\left(z_{k}\right)}\right] \mathrm{e}^{\mathrm{i} \theta_{k}}\right\}_{\mathrm{A}}^{\mathrm{A}^{*}}
\end{aligned}
$$


The displacement can be expressed as

$$
\begin{aligned}
2 \mu[u(z)+\mathrm{i} v(z)] & =2 \mu\left[u_{0}(z)+\mathrm{i} v_{0}(z)\right]+\sum_{k=1}^{N} 2 \mu\left[u_{k}\left(z_{k}\right)+\mathrm{i} v_{k}\left(z_{k}\right)\right] \mathrm{e}^{\mathrm{i} \theta_{k}} \\
& =\kappa \phi_{0}(z)-\omega_{0}(\bar{z})-(z-\bar{z}) \overline{\Phi_{0}(z)}+\sum_{k=1}^{N}\left[\kappa \phi_{k}\left(z_{k}\right)-\omega_{k}\left(\overline{z_{k}}\right)-\left(z_{k}-\overline{z_{k}}\right) \overline{\Phi_{k}\left(z_{k}\right)}\right] \mathrm{e}^{\mathrm{i} \theta_{k}}
\end{aligned}
$$

where

$$
\begin{gathered}
\phi_{0}(z)=\sum_{n=0,1}^{\infty} b_{n} z^{n}, \quad \omega_{0}(z)=\sum_{n=0,1}^{\infty} c_{n} z^{n} \\
\phi_{k}\left(z_{k}\right)=\omega_{k}\left(z_{k}\right)=-\sum_{m=1}^{\infty} \frac{a_{k}}{m} \alpha_{k m}\left(\frac{z_{k}}{a_{k}}-\sqrt{\frac{z_{k}^{2}}{a_{k}^{2}}-1}\right)^{m}-\alpha_{k 0} a_{k} \ln \left(\frac{z_{k}}{a_{k}}-\sqrt{\frac{z_{k}^{2}}{a_{k}^{2}}-1}\right)
\end{gathered}
$$

Equations (10) and (11) are the governing equations for determining the unknown coefficients $\alpha_{k m}(k=1,2, \ldots, N ; m=0,1,2, \ldots, \infty), b_{n}(n=0,1, \ldots, \infty)$ and $c_{n}(n=0,1, \ldots, \infty)$.

It is difficult to solve the governing equations analytically. The governing equations can be reduced to a system of linear algebraic equations for the unknown coefficients based on the boundary collocation method on crack surfaces and the outer boundary of the rectangular plate. Dividing the $k$-th crack surface into $M_{k}$ elements, the collocation points on the $k$-th crack surface are given by the following expression

$$
x_{k i}=a_{k} \cos \left(\frac{\mathrm{i} \pi}{M_{k}+1}\right), \quad i=1,2, \ldots, M_{k}
$$

The $i$-th outer edge of the rectangular plate is divided regularly into $N_{i}(i=1,2,3,4)$ segments by selecting the boundary stations $Q_{j}\left(z_{j}\right)(j=1,2, \ldots, N B)$, where $N B=\Sigma_{i=1}^{4} N_{i}, Q_{1}, Q_{2}$ and $Q_{N B}$ are taken as shown in Fig. 2.

When the algebraic equations are solved, the complex potentials and stress components produced by each crack, and the loads applied to the outer edges are known. According to the superposition principle, the stress fields of the rectangular plate are obtained with the aid of the transformation formulae from the local coordinate systems into the global one.

\section{SIFs and the average strains and stresses}

The stress intensity factors are related to stresses on the prolongation of the small cracks by

$$
K_{1 l}^{ \pm}-\mathrm{i} K_{2 l}^{ \pm}=\lim _{x_{l} \rightarrow \pm a_{l}} \sqrt{\pi} \sqrt{\frac{x_{l}^{2}-a_{l}^{2}}{a_{l}}}\left[\sigma_{y l}\left(x_{l}\right)-\mathrm{i} \sigma_{x y l}\left(x_{l}\right)\right] \quad\left|x_{l}\right|>a_{l}, \quad(l=1,2, \ldots, N)
$$

and they can be expressed further as

$$
K_{1 l}^{ \pm}-\mathrm{i} K_{2 l}^{ \pm}= \pm 2 \sqrt{\pi a_{l}} \sum_{m} \alpha_{l m} T_{m}( \pm 1), \quad l=1,2, \ldots, N
$$

in which the quantities with upper and lower signs refer to the right- and left-hand small crack tips, respectively.

Average stresses are defined in terms of quantities on the outer boundary $\Gamma$ [17]

$$
\langle\boldsymbol{\sigma}\rangle=\frac{1}{A} \int_{A} \boldsymbol{\sigma} \mathrm{d} A=\frac{1}{A} \int_{\Gamma} \mathbf{x t}^{0} \mathrm{~d} \Gamma
$$


where $\mathbf{x}$ is a position vector and $\mathbf{t}^{0}$ is a traction vector. $\mathbf{t}^{0}=\mathbf{n} \cdot \sigma, \sigma$ are the stresses of the outer boundary.

According to the work of Kachanov [5], for flat cracks in 2D, the average strains can be expressed as:

$$
\begin{aligned}
\langle\boldsymbol{\varepsilon}\rangle & =\mathbf{M}^{0}:\langle\boldsymbol{\sigma}\rangle+\frac{1}{2 A} \sum_{l}(\langle\boldsymbol{\delta}\rangle \mathbf{n}+\mathbf{n}\langle\boldsymbol{\delta}\rangle)^{l} a^{l} \\
& =\left(\mathbf{M}^{0}+\Delta \mathbf{M}\right):\langle\boldsymbol{\sigma}\rangle=\mathbf{M}:\langle\boldsymbol{\sigma}\rangle
\end{aligned}
$$

where $\mathbf{M}^{0}$ is the compliance tensor of the matrix material, $\langle\boldsymbol{\sigma}\rangle$ is the average stress, $\mathbf{M}$ is the effective compliance, $A$ is the area of the rectangular plate, the superscript $(l)$ denotes the $l$-th small crack in the plate, the summation is over all small cracks, and $a^{l}$ is the half-length of the $l$-th crack. $(\langle\boldsymbol{\delta}\rangle \mathbf{n})^{l},\left(\mathbf{n}\langle\boldsymbol{\delta}\rangle^{l}\right.$ denote dyadic (tensor) products of the displacement discontinuity vector $\boldsymbol{\delta}^{(l)}=$ $\mathbf{u}^{(l)+}-\mathbf{u}^{(l)-}$ and unit normal $\mathbf{n}^{(l)}$ to the $l$-th crack.

For a single crack, due to the closure condition at crack tips, the following equation should be satisfied

$$
\int_{-a}^{a} b(s) \mathrm{d} s=\frac{\mathrm{i} a(\kappa+1)}{\mu} \sum_{m=0}^{\infty} \alpha_{m} \int_{-1}^{1} \frac{T_{m}(\xi) \mathrm{d} \xi}{\sqrt{1-\xi^{2}}}=0
$$

According to the orthogonality of Chebyshev polynomials of the first kind, it is easily shown that

$$
\alpha_{0}=0
$$

The average opening and sliding displacements of the $l$-th crack can be calculated by the following formula:

$$
\langle\boldsymbol{\delta}\rangle^{l}=\frac{1}{2 a_{l}} \int_{-a_{l}}^{a_{l}} \boldsymbol{\delta}^{l} \mathrm{~d} x_{l}=\frac{a_{l} \pi(\kappa+1)}{4 \mu} \alpha_{l 1} \mathrm{i}
$$

For a bent crack, since the displacement should be single-valued, the following equation is obtained:

$$
\sum_{k=1}^{N_{j}} \alpha_{k 0} \mathrm{e}^{\mathrm{i} \theta_{k}}=0
$$

where $N_{j}$ is the number of bent cracks.

\section{EVOLUTION PROCESS OF SMALL CRACKS}

In this paper, the small crack evolution process is simulated based on linear elastic fracture mechanics. Two types of small crack growth are considered as intergranular growth and transgranular growth. For simplicity, new small crack nucleation is not considerd in this paper, but can easily be treated by the present method. Classical fracture mechanics criteria can be used as tools to determine whether an initial small crack will be activated. All small cracks are assumed to be open in this paper.

\section{Evolution process of small cracks}

Figure 3 shows an experimental uniaxial tensile stress-displacement curve of a concrete specimen given by Shah, Swartz and Onyang [18]. According to these results, a stress-strain curve can be divided into four stages. The first stage is before point A. All small cracks are stable and of initial size. No damage evolves in the material. Since all small cracks are open and randomly oriented, 


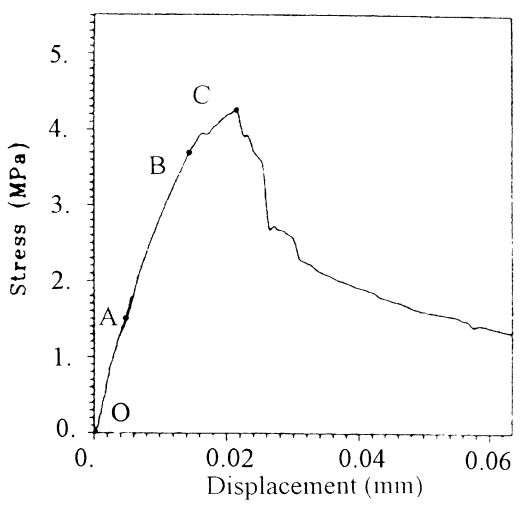

Fig. 3. A stress-displacement curve of concrete in tension and different cracking stages [18] (OA: stable cracks, AB: cracks initiate and propagate; $\mathrm{BC}$ : strain localization).

the overall response is isotropic and linear elasticity. The second stage is from point A to point B. Experimental observation [18] shows that the small cracks initiate and propagate in this stage. But these small cracks are isolated and randomly distributed over the specimen volume in the second stage. Intergranular growth and transgranular growth of pre-existing small cracks are simulated in this paper. The effect of grain boundary is indirectly considered by setting the critical stress intensity factors $K_{\text {ICC }}$ in transgranular growth. When the stress intensity factors of a preexisting small crack satisfy the fracture criterion of intergranular growth, this small crack becomes unstable and grows within the grain. For intergranular growth, the small crack growth is assumed to be in a self-similar fashion in its own direction, increasing its length from $2 a_{0}$ to $2 a_{u}$, where $2 a_{0}$ denotes the length of an initial small crack and $2 a_{u}$ signifies the grain size. With an increase in applied displacement, more and more small cracks satisfy the fracture criterion of intergranular growth and grow within the grain. The length of pre-existing small cracks can either be $2 a_{0}$ if the small cracks are stationary or $2 a_{u}$ if the small cracks are activated. In this stage, a few small cracks may satisfy the fracture criterion of transgranular growth and experience transgranular growth. According to the small crack geometries and distribution of stresses, the small crack can kink from the crack tip in the direction $\theta=\theta_{\mathrm{c}}$ and the length per transgranular growth is $2 a_{u}$, or the small crack links with the neighbour small crack. The third stage is between points B and C. The main feature of this stage is that the small cracks start to localize into a major crack that propagates with increasing load. New small cracks may nucleate, and pre-existing small cracks may grow to form a major crack in this stage. This phenomenon is referred to as damage localization. The crack propagation is stable until the peak load, where the stable crack growth means that the crack propagates only when the load increases. In our computation, a small crack that has undergone transgranular growth several times, will form a main crack in this stage. The stress-strain relation is evidently non-linear. The fourth stage is after the peak load. The major crack continuously propagates. The tensile strain of the material within the localized damage band increases, whereas unloading may occur for the material outside the damage band. In the present work, with a further increase in imposed displacement, the major crack will propagate further and other small cracks will experience unloading at the same time. The same fracture mechanics criterion is used in transgranular growth of small cracks and the growth of major cracks. Therefore, strain softening is a continuation of damage localization. 
Fracture criteria for small crack growth

\section{Intergranular growth}

For an open small crack, the following fracture criterion is employed for intergranular growth [19]:

$$
\left(\frac{K_{\mathrm{I}}}{K_{\mathrm{IC}}}\right)^{2}+\left(\frac{K_{\mathrm{II}}}{K_{\mathrm{IIC}}}\right)^{2}=1
$$

where $K_{\mathrm{I}}$ and $K_{\mathrm{II}}$ are the Mode I and II stress intensity factors, and $K_{\mathrm{IC}}$ an $K_{\mathrm{IIC}}$ are the Mode I and II critical stress intensity factors, respectively. The stress intensity factors of each small crack can be computed by Eq. (14). $K_{\mathrm{I}}$ and $K_{\mathrm{II}}$ depend on the exact small crack geometries, e.g. the number of small cracks, relative centre locations, relative spacing, relative orientations, etc.

When the local stress intensity factors of the small crack satisfy Eq. (22) for the weak plane, the microcrack becomes unstable and grows within the grain until reaching the grain boundary.

\section{Transgranular growth}

The maximum circumferential stress criterion $\left(\sigma_{\theta}\right.$-criterion) [20] is used for transgranular growth. This criterion may be stated as:

(i) The crack propagates along the direction $\theta=\theta_{\mathrm{c}}$ in which $\sigma_{\theta}$ becomes a maximum.

(ii) The crack extension starts when the stress intensity factor $K_{1}^{*}$ along the direction $\theta=\theta_{\mathrm{c}}$ reaches a critical value $K_{\mathrm{ICC}}$. The criterion condition of crack transgranular growth is given as:

$$
K_{\mathrm{I}}^{*}=K_{\mathrm{ICC}}
$$

where

$$
K_{\mathrm{I}}^{*}=\cos \frac{\theta_{\mathrm{c}}}{2}\left(K_{\mathrm{I}} \cos ^{2} \frac{\theta_{\mathrm{c}}}{2}-\frac{3}{2} K_{\mathrm{II}} \sin \theta_{\mathrm{c}}\right)
$$

$K_{\mathrm{ICC}}$ is the critical stress intensity factor for transgranular growth.

Once a small crack or main crack satisfies criterion (23), the small crack (or the main crack) will overcome the barrier of the grain boundary and penetrate into the neighbour grain.

\section{NUMERICAL EXAMPLES}

\section{$A$ bent crack in an infinite plate}

As a first example, consider an infinity plate containing a bent crack with an inclined angle of $45^{\circ}$ under uniform tension, as shown in Fig. 4. The normalized stress intensity factors are given in Table 1, along with the results by Isida [21]. Table 1 shows that the results of the present method agree well with Isida's results [21].

For a skew-symmetric bent crack (as shown in Fig. 5) under remote tension loading, the normalized stress intensity factors are shown in Table 2, which agree well with Kitagawa and Yuuki's solutions [21].

Numerical tests have shown that the accuracy and efficiency of the present method are very high, so this method can be used as a tool to study the damage problems of brittle materials weakened by a large number of cracks. 


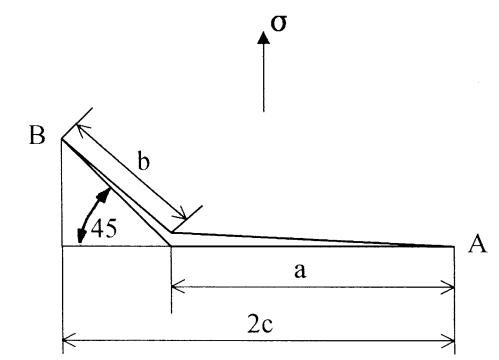

Fig. 4. A bent crack under uniform tension.

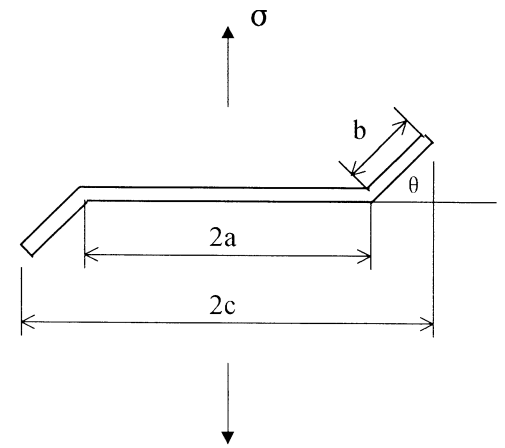

Fig. 5. A skew-symmetric bent crack under uniform tension.
Table 1. Normalized stress intensity factors for the geometry of Fig. 4

\begin{tabular}{|c|c|c|c|c|c|c|c|c|}
\hline \multirow[b]{2}{*}{$b / a$} & \multicolumn{2}{|c|}{$K_{\mathrm{I}}^{\mathrm{A}} / \sigma \sqrt{\pi c}$} & \multicolumn{2}{|c|}{$K_{\mathrm{II}}^{\mathrm{A}} / \sigma \sqrt{\pi c}$} & \multicolumn{2}{|c|}{$K_{\mathrm{I}}^{\mathrm{B}} / \sigma \sqrt{\pi c}$} & \multicolumn{2}{|c|}{$-K_{\mathrm{II}}^{\mathrm{B}} / \sigma \sqrt{\pi c}$} \\
\hline & $\begin{array}{c}\text { Present } \\
\text { results }\end{array}$ & $\begin{array}{l}\text { Isida } \\
{[21]}\end{array}$ & $\begin{array}{l}\text { Present } \\
\text { results }\end{array}$ & $\begin{array}{l}\text { Isida } \\
{[21]}\end{array}$ & $\begin{array}{l}\text { Present } \\
\text { results }\end{array}$ & $\begin{array}{l}\text { Isida } \\
{[21]}\end{array}$ & $\begin{array}{l}\text { Present } \\
\text { results }\end{array}$ & $\begin{array}{l}\text { Isida } \\
{[21]}\end{array}$ \\
\hline 01 & & 1.00 & & & & 0.7 & & 0.3 \\
\hline & & 1 & & & & 0.66 & & 0.4 \\
\hline & & & & & & & & 0.504 \\
\hline & & & & & & & & 0.55 \\
\hline 4 & 0. & 0 & & & & 0.574 & & 0.60 \\
\hline & & & & & & 0. & & 0.619 \\
\hline & & & & & & & & 067 \\
\hline & & & & & & 0. & & 0.637 \\
\hline & & & 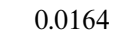 & & & 0.569 & & 0.641 \\
\hline & & & & & & 0574 & & \\
\hline 2.0 & 0.9932 & 0.993 & -0.022 & -0.022 & 0.5789 & 0.579 & 0.6384 & 0.63 \\
\hline
\end{tabular}

Table 2. Normalized stress intensity factors for the geometry of Fig. 5 $\left(F_{\mathrm{I}}=K_{\mathrm{I}} / \sigma \sqrt{\pi c}, F_{\mathrm{II}}=K_{\mathrm{II}} / \sigma \sqrt{\pi c}, \theta=45^{\circ}\right)$

\begin{tabular}{llccccccc}
\hline \multicolumn{1}{c}{$b / a$} & 0.05 & 0.1 & 0.2 & 0.4 & 0.6 & 0.8 & 1.0 \\
\hline$F_{\text {I }}$ & $\begin{array}{l}\text { Present results } \\
\text { Results of }\end{array}$ & 0.7052 & 0.6804 & 0.6530 & 0.6277 & 0.6156 & 0.6091 & 0.6051 \\
& & & & & & & \\
& Kitagawa and \\
& $\begin{array}{l}\text { Yuuki [21] } \\
F_{\text {II }}\end{array}$ & 0.7058 & 0.6805 & 0.6532 & 0.6280 & 0.6161 & 0.6095 & 0.6054 \\
& $\begin{array}{l}\text { Present results } \\
\text { Results of }\end{array}$ & 0.4180 & 0.4501 & 0.4884 & 0.5289 & 0.5486 & 0.5616 & 0.5699 \\
& & & & & & & \\
Kitagawa and & & & & & & & \\
& Yuuki [21] & 0.4184 & 0.4507 & 0.4888 & 0.5284 & 0.5491 & 0.5617 & 0.5698 \\
\hline
\end{tabular}

Uniform displacement of centre-cracked rectangular plate

A rectangular plate with a centre crack is subjected to uniform displacement without shear, as shown in Fig. 6. The number of collocation points on the crack surface is 8 , and the number of collocation points of the outer boundary is $60(N B=60)$. The normalized stress intensity factors are given in Table 3, which are in good agreement with Isida's solutions [21]. The results show the present method is efficient for solving the crack problem of a finite solid.

\section{Uniform displacement of rectangular plate with small cracks}

In the present work, crack density is the parameter that characterizes the effect of small cracking. The crack density is defined for a small cracked solid (2D) as [1]

$$
\rho=\frac{1}{A} \sum_{i=1}^{N} a_{i}^{2}
$$

where $N$ is the number of small cracks, $a_{i}$ is the half-length of the $i$-th small crack, and $A$ is the area of the rectangular plate. The present study is limited to the plane stress analysis.

In this paper, 25 randomly oriented small cracks with the same length are generated in the square plate. The square plate is divided uniformly into meshes and each mesh contains one small 


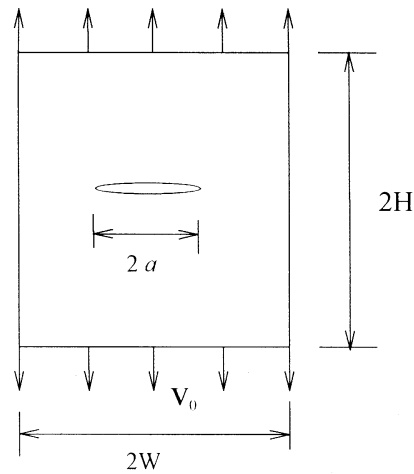

Table 3. Values of $K_{\mathrm{I}} /\left[\sqrt{\pi} E v_{0} /(\pi H)^{1 / 2}\right]$ for the geometry of Fig. $6(H / W=1)$

\begin{tabular}{lllllllll}
\hline \multicolumn{1}{c}{$a / W$} & 0.1 & 0.2 & 0.3 & 0.4 & 0.5 & 0.6 & 0.7 & 0.8 \\
\hline Present & 0.5544 & 0.7594 & 0.8862 & 0.9639 & 1.0093 & 1.0316 & 1.0438 & 1.055 \\
Isida [21] & 0.554 & 0.759 & 0.886 & 0.964 & 1.009 & 1.032 & 1.042 & 1.05 \\
\hline
\end{tabular}

$\mathrm{H}$

Fig. 6. Uniform displacement without shear of centre-cracked rectangular plate.

crack. Locations and orientation of small cracks are randomly generated in each mesh [Fig. 7(a)]. In the course of generation, they are regenerated if there is an intersection among the cracks, or an intersection between small cracks and the boundary. In order to illustrate the stress-strain relation of a square plate containing randomly oriented small cracks, following the work done by $\mathrm{Ju}$ and Lee [8], we take $K_{\mathrm{IC}}=0.2592 \mathrm{MN} / \mathrm{m}^{3 / 2}, K_{\mathrm{IIC}}=0.51836 \mathrm{MN} / \mathrm{m}^{3 / 2}, K_{\mathrm{ICC}}=0.58 \mathrm{MN} / \mathrm{m}^{3 / 2}$, $a_{0}=0.196 \mathrm{~cm}, a_{u}=0.49 \mathrm{~cm}, E=34450 \mathrm{MPa}, v=0.3, H=W=3.8 \mathrm{~cm}$. Four sample arrays are considered. The damage evolution process of a square plate containing multiple small cracks is given as follows: for sample 1 , when the tensile stress $\sigma \leqslant \sigma_{\mathrm{c}}\left(\sigma_{\mathrm{c}}\right.$ is the critical stress of damage occurring), all small cracks are stable and of initial size [Fig. 7(a)], the overall stress-strain relation is linear elastic. When $\sigma$ reaches $\sigma_{\mathrm{c}}$, a small crack which satisfied Eq. (22) will grow within the grain and its size becomes $2 a_{u}$. With the increase of applied displacement, more and more small cracks satisfy the fracture criterion Eq. (22) and grow within the grain [Fig. 7(b)]. With the further increase in imposed displacement, transgranular growth occurs. If the small crack, having size $2 a_{u}$, satisfies criterion (23), it will experience transgranular growth. With the further increase in imposed displacement, the small crack that has undergone transgranular growth will propagate further to localize into a major crack. The stress-strain relation exhibits an evidently non-linear behaviour. A major crack continuously propagates [Fig. 7(c)], and a softening-type stress-strain curve is obtained. Figure 8 displays the SEM micrograph of an actual $\mathrm{Si}_{3} \mathrm{~N}_{4}$ ceramics specimen. From a

(a)

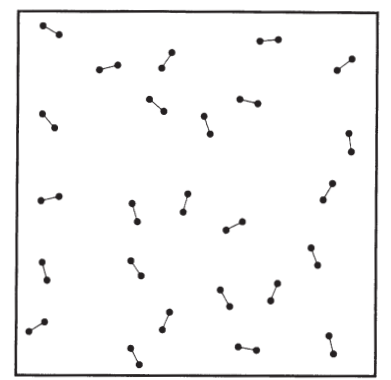

(b)

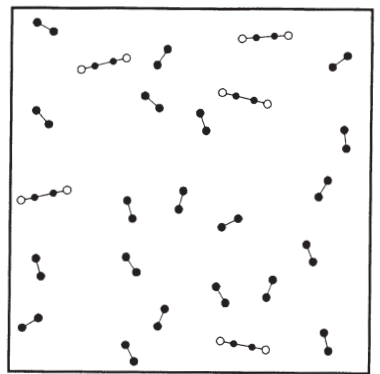

(c)

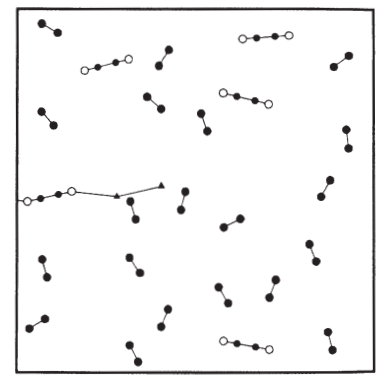

Fig. 7. Evolutionary damage process of 25 small cracks in a square plate (sample 1$)(H=W=3.8 \mathrm{~cm}$, $a_{0}=0.196 \mathrm{~cm}, a_{u}=0.49 \mathrm{~cm} ; \boldsymbol{O}=$ pre-existing small cracks, $\bigcirc=$ small cracks after intergranular growth, $\boldsymbol{\Delta}=$ small cracks after transgranular growth). 


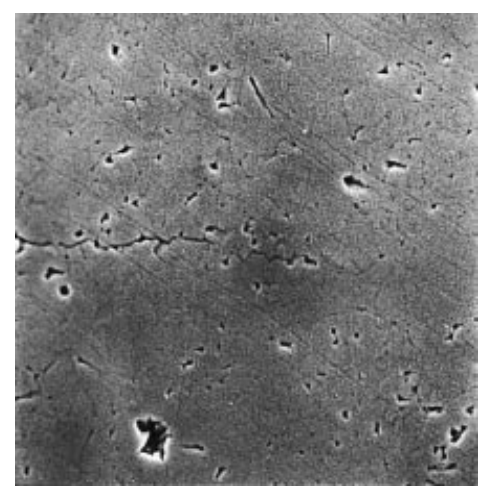

Fig. 8. A SEM micrograph of $\mathrm{Si}_{3} \mathrm{~N}_{4}$ ceramics over an area of $18 \times 18$ microns.

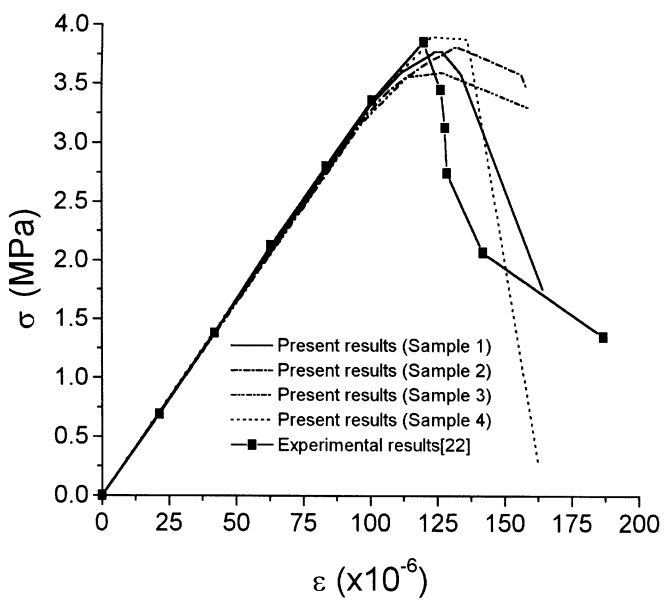

Fig. 9. The stress-strain curves.

comparison of the results, it can be found that the damage evolution process of a square plate containing multiple small cracks is consistent with the experimental result. The stress-strain relation curves of four smples are given in Fig. 9, along with the experimental results of Gopalaratnam and Shah [22]. Figure 9 shows that the present results have scatter from one sample to another that really reflects the features of randomly distributed cracks. It can be seen that the present results are in agreement with the experimental results [22]. The results show that the present micromechanical damage theory can study the damage process of multiple small cracks in solids.

\section{CONCLUSIONS}

A micromechanical damage theory has been presented in this paper which accounts for the interactions between different small cracks and the effect of the boundary of a finite plate. The evolution processes of small cracks are simulated, and the stress-strain relation curves for a finite plate with small cracks are given. The numerical results show that the present method is accurate and efficient for evaluating the SIFs of multiple cracks (including a bent crack), and the simulated damage evolution process for a square plate containing multiple small cracks is consistent with the experimental results. Thus, the present theory can be used to study the damage process of brittle materials.

Acknowledgements-The research presented here was supported by the National Natural Science Foundation of China (no. 19704100) and the National Natural Science Foundation of Chinese Academy of Sciences (project KJ951-1-201). The authors are thankful to Prof. N. G. Liang and Mr W. D. Yan for the SEM micrograph of $\mathrm{Si}_{3} \mathrm{~N}_{4}$ ceramics specimen.

\section{REFERENCES}

1. B. Budiansky and R. J. O’Connell (1976) Elastic moduli of a cracked solid. Int. J. Solids Structures 12, 81-95.

2. R. M. Christensen and K. H. Lo (1979) Solutions for effective shear properties in three phase sphere and cylinder models. J. Mech. Phys. Solids 27, 315-330.

3. Z. Hashin (1988) The differential scheme and its application to cracked materials. J. Mech. Phys. Solids 36, 719-734. 
4. T. Mori and K. Tanaka (1973) Average stress in matrix and average elastic energy of materials with misfitting inclusions. Acta Metall. 21, 571-574.

5. M. Kachanov (1992) Effective elastic properties of cracked solids: critical review of some basic concepts. Appl. Mech. Rev. 45, 304-335.

6. Y. Huang, A. Chandra, Z. Q. Jiang, X. Wei and K. X. Hu (1996) The numerical calculation of twodimensional effective moduli for microcracked solids. Int. J. Solids Structures 33, 1575-1586.

7. D. Krajcinovic and D. Fanella (1986) A micromechanical damage model for concrete. Engng Fracture Mech. 25(5/6), 585-596.

8. J. W. Ju and X. Lee (1991) Micromechanical damage models for brittle solids. I: Tensile loadings. J. Engng Mech. 117, 1495-1514.

9. M. Ortiz (1988) Microcrack coalescence and macroscopic crack initiation in brittle solids. Int. J. Solids Structures 24, 231-250.

10. X. Huang and B. L. Karihaloo (1992) Tension softening of quasi-brittle materials modelled by single and doubly periodic arrays of coplanar penny-shaped cracks. Mech. Mater. 13, 257-275.

11. H. Horii, A. Hasegawa and F. Nishino (1989) Fracture process and bridging zone model and influencing fracture of concrete. In: Fracture of Concrete and Rock (Edited by S. Shah and S. Swartz), Springer, New York, pp. 205-209.

12. W. F. Chen and E. Yamaguchi (1990) Micromechanics of Failure of Quasi-brittle Materials, Elsevier Applied Science, London, pp. 165-274.

13. X. Q. Feng and S. W. Yu (1995) Micromechanical modelling of tensile response of elastic-brittle materials. Int. J. Solids Structures 32, 3359-3372.

14. N. I. Muskhelishvilli (1953) Some Basic Problems of Mathematical Theory of Elasticity, P. Noordhoff, Groningen.

15. D. Gross (1982). Spannungsintensitätsfaktoren von ribsystemen. Ing. Arch. 51, 301-310.

16. X. L. Han and T. C. Wang (1996) Elastic fields of arbitrarily and periodically distributed cracks. In: Progress in Advanced Materials and Mechanics (Edited by T. C. Wang and T. W. Chou), Peking University Press, Beijing, pp. 688-693.

17. S. Nemat-Nasser and M. Hori (1993) Micromechanics: Overall Properties of Heterogeneous Materials, Elsevier Science, North-Holland.

18. S. Shah, S. E. Swartz and C. S. Ouyang (1995) Fracture Mechanics of Concrete: Applications of Fracture Mechanics to Concrete, Rock, and Other Quasi-Brittle Materials, Wiley, New York.

19. M. F. Kanninen and C. H. Popelar (1985) Advanced Fracture Mechanics, Oxford University Press, New York.

20. F. Erdogan and G. C. Sih (1963) On the crack extension in plates under plane loading with transverse shear. J. Basic Engng ASME, Ser. D 85, 519-527.

21. Y. Murakami (1987) Stress Intensity Factors Handbook, World Publishing, Pergamon Press.

22. V. S. Gopalaratnam and S. P. Shah (1985) Softening response of plain concrete in direct tension. $A C I$ Journal, 310-323. 